\title{
SegNet Mimarisi Kullanılarak Cilt Lezyon Bölütleme Performansının İyileştirilmesi*
}

\author{
Nurullah Şahin ${ }^{1}$, Nuh Alpaslan ${ }^{2}$ \\ 1 İnönü Üniversitesi, Mühendislik Fakültesi, Malatya, Türkiye (ORCID: 0000-0002-3578-9959) \\ ${ }^{2}$ Bingöl Üniversitesi, Mühendislik ve Mimarlık Fakültesi, Bingöl, Türkiye (ORCID: 0000-0002-6828-755X)
}

(Konferans Tarihi: 5-7 Mart 2020)

(DOI: 10.31590/ejosat.araconf6)

ATIF/REFERENCE: Şahin, N. \& Alpaslan, N. (2020). SegNet Mimarisi Kullanılarak Cilt Lezyon Bölütleme Performansının İyileştirilmesi. Avrupa Bilim ve Teknoloji Dergisi, (Özel Sayı), 40-45.

$\ddot{\mathbf{O} z}$

Kötü huylu melanom, bütün cilt kanseri türleri arasında üçüncü en sık rastlanan tür olmasına rağmen en çok ölüme neden olan formudur. Kötü huylu melanomun erken aşamada teşhisi hastanın yaşama şanşını büyük oranda artırdığından, erken teşhis oldukça önemlidir. Melanom teşhisi dermatologlar tarafından lezyon bölgesinin geometrisi, rengi, yapısal ve dokusal özellikleri gibi görsel niteliklerine bakılarak yapılmaktadır. Ancak, son zamanlarda, bilgisayarlı görü ve makine öğrenmesi yöntemlerindeki gelişmeler ile birlikte melanoma tanısı için bilgisayar destekli tanı sistemleri popüler olmaya başlamıştır. Bu çalışmada ciltte bulunan lezyonların bölütlenmesi için SegNet mimarisi tabanlı bir sistem geliştirilmiş̧ir. Bunun yanında, cilt lezyonları üzerinde veri büyütme ve renk tutarlılı̆ı ve kıl silme gibi önişleme adımların bölütleme performansı üzerinde etkileri incelenmiştir. Deneylerimizde ISBI2016 veri kümesi kullanılmıştır. Sonuçlar veri büyütme ve önişlemenin bölütleme performasını dikkate değer oranda artırdığını göstermektedir. Bununla birlikte, veri büyütmenin ezberlemeyi önlediği ve modellerin genelleme yeteneğini artırdığı sonucuna varılmıştır.

Anahtar Kelimeler: melanoma, bölütleme, derin öğrenme, lezyon, segnet.

\section{Improving Skin lesion Segmentation Performance Using SegNet Architecture}

\begin{abstract}
The malignant melanoma is the third most common form of skin cancer among all skin cancer types, but it is the most fatal form of skin cancer. Early diagnosis is very important, as the early diagnosis of malignant melanoma greatly increases the patient's survival chance. The melanoma diagnosis is carried out by dermatologists by examining the visual characteristics of the lesion area such as geometry, color, structural and textural features. However, recently, the computer-aided diagnosis systems have become popular for the melanoma detection, with advances in computer vision and machine learning methods. In this study, a SegNet architecture based system has been developed for segmentation of skin lesions. In addition, the effects of preprocessing steps on skin lesions such as data augmentation and color consistency and hair removal were investigated on segmentation performance. ISBI2016 dataset was used in our experiments. The results show that data augmentation and preprocessing significantly increases segmentation performance. However, it was concluded that data augmentation prevents memorization and increases the generalization ability of the models.
\end{abstract}

Keywords: Melanoma, Segmentation, Deep Learning, Skin Lesion, SegNet.

\footnotetext{
* Bu makale International Conference on Access to Recent Advances in Engineering and Digitalization (ARACONF 2020) de sunulmuştur.
} 


\section{Giriş}

Melanom, cilt kanserleri türleri içinde en sık görülen üçüncü cilt kanseri türü olmakla beraber, cilt kanserleri türleri içerisinde en ölümcül olanıdır (Burg, 1993) (Schmid-Saugeon, Guillod ve Thiran, 2003). Cilt kanseri gelişmiş ve gelişmekte olan ülkelerde son yıllarda oldukça sık görülen kanser türlerinin başında gelmektedir (L.A.G. Ries, 2008). Amerika Birleşik Devletleri'nde her yıl yaklaşık 76.380 yeni melanom vakası görülmekte ve tahmini 6.750 kişi hayatını kaybetmektedir (L.A.G. Ries, 2008).

Kötü huylu melanomda hayatta kalma şansı, büyük oranda erken tanı ve doğru ve teşhise bağlıdır (Romero Lopez, Giro-INieto, Burdick ve Marques, 2017). Literatürde yapılan çalışmalar incelendiğinde, erken teşhis ile melanom \%98 oranında tedavi edilebilmektedir. Bununla birlikte, günümüzde melonum \%65-\%80 oranında doğru teşhis edilebilmektedir (Argenziano ve Soyer, 2001). Bu oran güncel bilgisayarlı görü ve makine öğrenmesi yöntemleri ile artırılabilir. Literatürde cilt kanserinin klinik tanısına yardımcı olmak için ABCD kuralı (Stolz, Reimann ve Cognetta, 1994), 7 noktalı kontrol listesi (Argenziano ve diğerleri, 1998) ve Menzies yöntemi (Menzies, Ingvar, Crotty ve McCarthy, 1996) gibi birçok teknik bulunmaktadır. Bu yöntemler içinde en yaygın kabul gören yöntem $\mathrm{ABCD}$ kuralıdır. $\mathrm{ABCD}$ skorunun tespitinde kritik ișlem kitle sınırlarının belirlenmesidir (Stolz ve diğerleri, 1994). Çünkü dermatoskopik cilt lezyonlarında bulunan kıl ve benzeri bölgeler kitlenin görülmesini engellemektedir. Bu nedenle, kitleli bölgenin kesin olarak belirlenmesi için, kıl gibi alanların kaldırılması gerekir. Dermatoskopik cilt lezyonlarındaki kitle sınırlarının belirlenmesinde yaygın olarak kullanılan yöntemler sırasıyla, eșikleme (Garnavi, Aldeen, Celebi, Varigos ve Finch, 2011), bölge genişleme (Erkol, Moss, Stanley, Stoecker ve Hvatum, 2005), kümeleme algoritmaları (Agarwal, Issac, Dutta, Riha ve Uher, 2017), Geodesic Aktif veya Bölge Tabanlı Contour modelleri, GAC (Kasmi, Mokrani, Rader, Cole ve Stoecker, 2016) ve RAC (Chan ve Vese, 2001), İstatistiki bölge tekniklerini birleştirme (Wong, Scharcanski ve Fieguth, 2011) olarak sıralanabilir. (Garnavi ve diğerleri, 2011) lezyon alanlarını belirlemek için hibrid eşik bazlı sınır saptama yöntemi uygulamıştır. (Erkol ve diğerleri, 2005), gradyan vektör akışı gibi aktif kontur yöntemlerine dayanan bir segmentasyon metodu sunmuşlardır. Daha güncel çalışmalarda (Bi ve diğerleri, 2016), çok ölçekli süper piksellere dayanan otomatik bir hücresel SLS yöntemi önermiştir. (Ahn ve diğerleri, 2017) görsel belirginliğe (saliency) dayalı dermoskopi görüntülerinde arka plan tespiti yaklaşımı ve bir cilt lezyon bölütleme yaklaşımı (Ahn ve diğerleri, 2015) önermiştir. Derin öğrenme mimarisindeki gelişmelere paralel olarak da (Xie ve Bovik, 2013), kendi kendini üreten sinir ağı ve genetik algoritmanın kombinasyonuna dayanan bir yöntem önermişlerdir. (Bi, Kim, Ahn, Feng ve Fulham, 2017) tam evrişimli sinir ağı kullanarak yarı otomatik bir cilt lezyonu bölütlemesi yöntemi önermişlerdir.

Cilt lezyon segmentasyonu için kullanılan geleneksel yöntemler belirli bir ölçüde başarılı olsalar bile, farklı kontrast değerleri, ve diğer sorunların tamamı ile baş etmekte sıkıntı yaşamaktadırlar. Bu nedenle son yıllarda grafik işlemci birimlerindeki gelişmeler ile derin öğrenme tabanlı yöntemler görüntü segmentasyonunda oldukça yaygın olarak kullanılmaya başlanmıştır. Peng Tang ve arkadaşları, ayrıştırılabilir evrişimsel bloklardan oluşan bir U-Net modeli önermiştir (Tang ve diğerleri, 2019). Prashant Brahmbhatt vd. ikili cross-entropy yöntemine dayanan bir SegNet modeli önermiştir (Brahmbhatt ve Rajan, 2019).

$\mathrm{Bu}$ çalışmada cilt lezyonları üzerinde veri çoklama ve renk tutarlılığı ve kıl silme gibi önişleme adımların bölütleme performansı üzerinde etkileri incelenmiştir. Kıl silme işlemi için DullRazor, renk sabitliği için shades of gray algoritmaları kullanılmıştır. Bu amaçla, öncelikle, RGB görüntü üzerinde hiçbir önişleme yapılmadan SegNet mimarisi kullanılarak bölütleme başarımı gözlemlenmiştir. Sonrasında, görüntülere yeniden boyutlandırma, kıl silme, ve görüntülerde renk tutarlılı̆̆nın sağlanabilmesi adına bir dizi önişleme adımları ve boşluk silme ve küçük alanların silinmesi gibi bir dizi sonişleme adımları uygulanmıştır. Sonuçlar ISBI2016 veri kümesi üzerinde farklı bölütleme ölçütleri kullanılarak test edilmiştir. Elde edilen sonuçlar güncel literatür ile kıyaslanmıştır. Elde edilen sonuçlar veri çoklama ve önişleme adımlarının bölütleme performasını dikkate değer oranda artırdığını göstermektedir. Ayrıca, veri çoklamanın ezberlemeyi (overfitting ) önlediği ve bu nedenle modellerin genelleme yeteneğini artırdığı sonucuna varılmıştır.

\section{Materyal ve Metot}

Cilt lezyonları için oluşturulan sınırı sayıda veri kümesi bulunmaktadır. Ayrıca bu veri setlerinin boyutları genellikle küçük olup bunların bir kısmı halka açık değildir. Cilt lezyon veri setleri olarak yaygın olarak kullanılan PH2, ISBI2016, ISBI2017 veri setleri gösterilebilir. Bu çalışmada ISBI2016 veri kümesi kullanılmıştır. Bu bölümde cilt lezyon bölütlemesi için kullanılan adımlardan bahsedilecektir. Cilt lezyonlarının bölütlenmesi için uygulanan adımlar Şekil 1'de gösterilmiştir. 


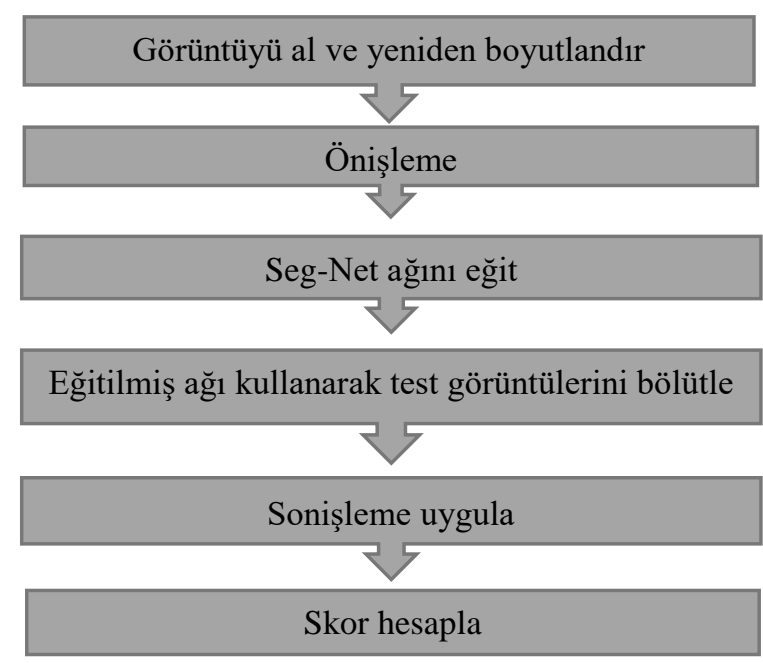

Şekil 1. Aklş Diagramı

İlk olarak SegNet mimarisi ile bölütleyeceğimiz görüntülerde, derin öğrenme mimarilerinin büyük boyutlu resimleri işlemeleri sırasında yaşanacak işlem hacmindeki aşırı artışı önlemek ve eğitim süresini kısaltmak için, görüntüler 192x256 şeklinde yeniden boyutlandırıldı. Sonrasında, kıl silme ve renk sabitleme (shades of gray) adımları uygulanmıştır. Son olarak boşluk doldurma ve küçük parçacıkların silinmesi adımları uygulanarak lezyonlu bölge belirginleştirilmiştir.

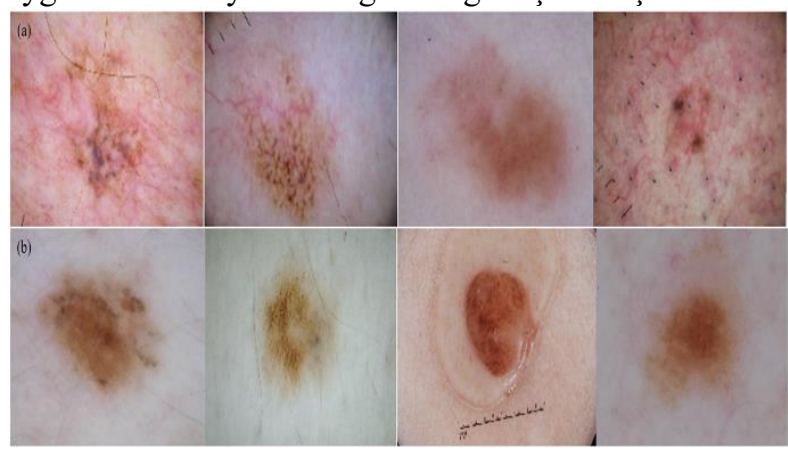

Şekil 2. ISBI 2016 veri kümesine ait (a) Melanom (b) Nevus görüntüleri.

Şekil 2'de iki farklı lezyon görülmektedir. Şekil 2(a)' da melanom lezyonlar görülmektedir. Şekil 2(b)' de ise melanom olmayan dermoskopik lezyonlar görülmektedir.

Bu çalışmada kıl silme işlemi için DullRazor yöntemi kullanılmıştır. DullRazor adından da anlaşılacağı üzere, özellikle kalın ve mat kılların silinmesinde oldukça etkili bir yöntem olup yöntemin eksi yanı çok ince kıllarda başarılı olmamasıdır. DullRazor Algoritması genel olarak; kılların konumlarını belirleme, kıl piksellerinin yakınındaki bir alandan saç olmayan piksellerle değiştirilmesi, ve değiştirilen bölgenin yumuşatılması aşamalarından oluşur (Lee, Ng, Gallagher, Coldman ve McLean, 1997).
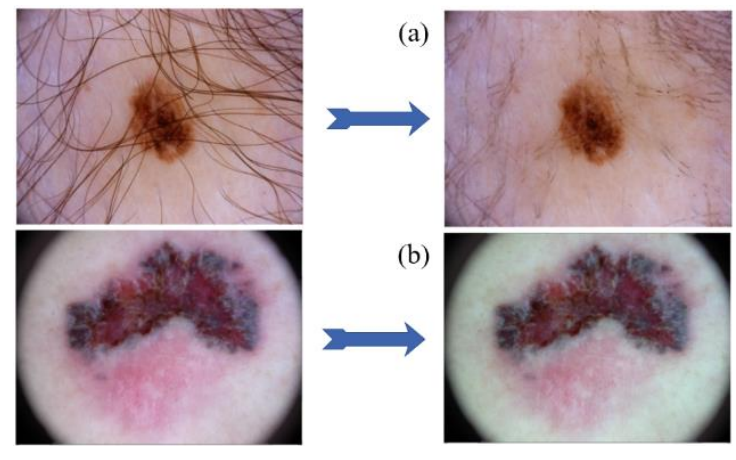

Şekil 3. (a) DullRazor ve (b) Shades of gray algoritmalarının sonuçları

Renk Sabitliği için shades of gray algoritması kullanılmıştır (Barata, Celebi ve Marques, 2015). Amaç, bilinmeyen bir ışık kaynağı kullanılarak edinilen bir görüntünün renklerini, standart bir 1şık kaynağı altındaki renklerle aynı görünecek şekilde dönüştürmektir. Bu şekilde daha tutarlı görüntüler elde etmek ve bölütlendirme başarısını arttırmak hedeflenmektedir. Şekil 3(a)'da DullRazor kıl silme Şekil 3(b)'de ise Shades of Gray algoritmasının sonuçları görülmektedir. 
SegNet sinir ağı, Cambridge Üniversitesi'nde geliştirilen, anlamsal piksel bilgi etiketleme veya daha genel tabirle anlamsal bölütleme için kullanılan evrişimli bir sinir ağıdır. Anlamsal segmentasyon için kullanılan ağlar RGB görüntüsünü girdi verisi olarak alır ve görüntüdeki $n$ adet nesne için $n$ adet etiket değerlerine sahiptir. Her nesne araba, yol vb. gibi bir etikete karşllık gelmektedir. Belirli bir nesnedeki her piksel, o pikselin o nesneye karşıllk gelen etikete ait olup olmamasına bağlı olarak 1 veya 0 olacaktır (Badrinarayanan, Kendall ve Cipolla, 2017).

SegNet, kodlayıcı ve kod çözücü olarak adlandırılan katmanlardan oluşur. Her kodlayıcı, bir adet evrişim bir adet BN(batch normalization-Topluluk Normalizasyonu) ve RELU adımların içerir. Kodlayıcı adımlarından çıkan değerler ardından bir maksimum havuzlama katmanında işlenir. Kod çözücüler temel olarak kodlayıcılara benzer. Farkları Doğrusal olmaları ve kodlama aşamasından saklanan endeksleri kullanarak girdilerini örneklendirmeleridir. Son kod çözücüden alınan çıktı daha sonra bir SoftMax sınıflandırıcısına verilerek bölütleme işlemi gerçekleştirilir. Seg-Net mimarisi Şekil 4 'te gösterilmiştir. Çalışmada kodlayıcı derinliği 4 (dört) olarak belirlenmiştir. Ağın öğrenme aşamasında öğrenme algoritması olarak adam optimizasyon yöntemi kullanılmıştır. Bölütleme katmanında çarpraz entropi yöntemi kullanılmıştır.

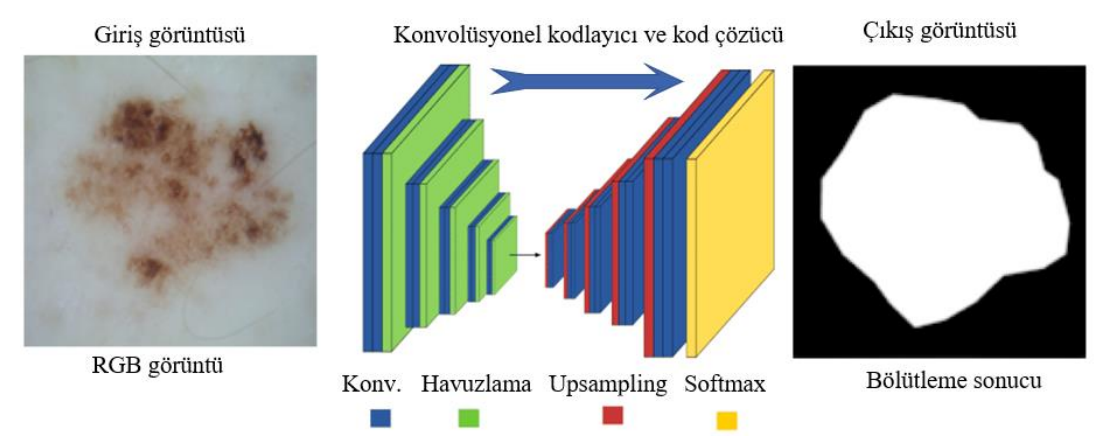

Şekil 4. SegNet mimarisi.

\section{Araştırma Sonuçları ve Tartışma}

$\mathrm{Bu}$ bölümde önerilen yöntem, 900 görüntüden oluşan ISBI2016 veri kümesine uygulanarak elde edilen sonuçlar değerlendirilmiştir. Eğitim setinde yer alan 900 görüntüden 630'u eğitim 270'i test amaçlı kullanılmıştır. İlk deneyde veri çoklama ve önişleme adımları kullanılmadan SegNet mimarisinin performansı değerlendirildi. İkinci adımda eğitim seti üzerinde veri çoklama uygulanarak sonuçlar değerlendirildi. Son adımda ise eğitim seti üzerinde veri çoklama ve tüm görüntüler üzerinde kıl silme ve renk sabitleme önişleme adımları uygulanarak sonuçlar değerlendirilmiştir.

Eğitim örneklerimizden en iyi şekilde yararlanmak ve modelin doğruluğunu artırmak için, veriler bir dizi rasgele dönüşümle çoklanmıştır. Veri çoklama aşamasında resimler $\pm 10^{0}$ döndürme, yatay ve dikey eksende kaydırma ve aynalama işlemlerine tabi tutuldu. Ayrıca, veri çoklama kullanarak ezberlemeyi (overfitting ) önlemek ve bu nedenle modellerin genelleme yeteneğini geliştirmek amaçlandı. Doğru öğrenme, yetersiz öğrenme ve aşırı öğrenme durumları Şekil 5’te gösterilmiştir.

Tablo 1. Bölütleme için performans kriterleri hesaplanması

\begin{tabular}{|c|c|}
\hline $\begin{array}{l}\text { Doğruluk (Accuracy): } \\
\qquad A C=\frac{D P+D N}{D P+Y P+D N+Y N}\end{array}$ & $\begin{array}{l}\text { Hassasiyet (Sensitivity): } \\
\qquad S E=\frac{D P}{D P+Y N}\end{array}$ \\
\hline $\begin{array}{l}\text { Özgüllük (Specificity): } \\
\qquad S P=\frac{D N}{D N+Y P}\end{array}$ & $\begin{array}{l}\text { Dice katsay1s1: } \\
\qquad D I=\frac{2 * D P}{2 * D P+Y P+Y N}\end{array}$ \\
\hline & $+Y N$ \\
\hline
\end{tabular}

Bölütleme performans değerlendirmesi için Tablo 1'de görülen ölçütler kullanılmıştır. Bu ölçütler piksel seviyesinde hesaplanmıştır. Burada, DP, gerçekte lezyon olan ve lezyon olarak belirlenen piksel sayısını; YN, gerçekte lezyon olan hatalı şekilde lezyon olmayan olarak belirlenen piksel sayısını; YP, gerçekte lezyon olmayan ve hatalı şekilde lezyon olarak belirlenen piksel sayısını; DN, gerçekte lezyon olmayan ve lezyon olmayan olarak belirlenen piksel sayısını ifade etmektedir. Doğruluk, doğru bir şekilde tahmin edilmiş örneklerin toplam örnek sayısına oranıdır. Özgüllük, doğru tahmin edilen negatiflerin toplam negatiflere 
oranıdır. Hassasiyet, doğru tahmin edilen pozitiflerin, toplam pozitif tahminlere oranıdır. Diğer iki ölçüt ise gerçek yara bölgesi ile tahmin edilen bölge arasındaki örtüşmeyi ölçen Dice katsayısı ve gerçek yara bölgesi ile tahmin edilen bölgelerin birleşimlerinin kesişimlerini ölçen Jaccard indeksidir.
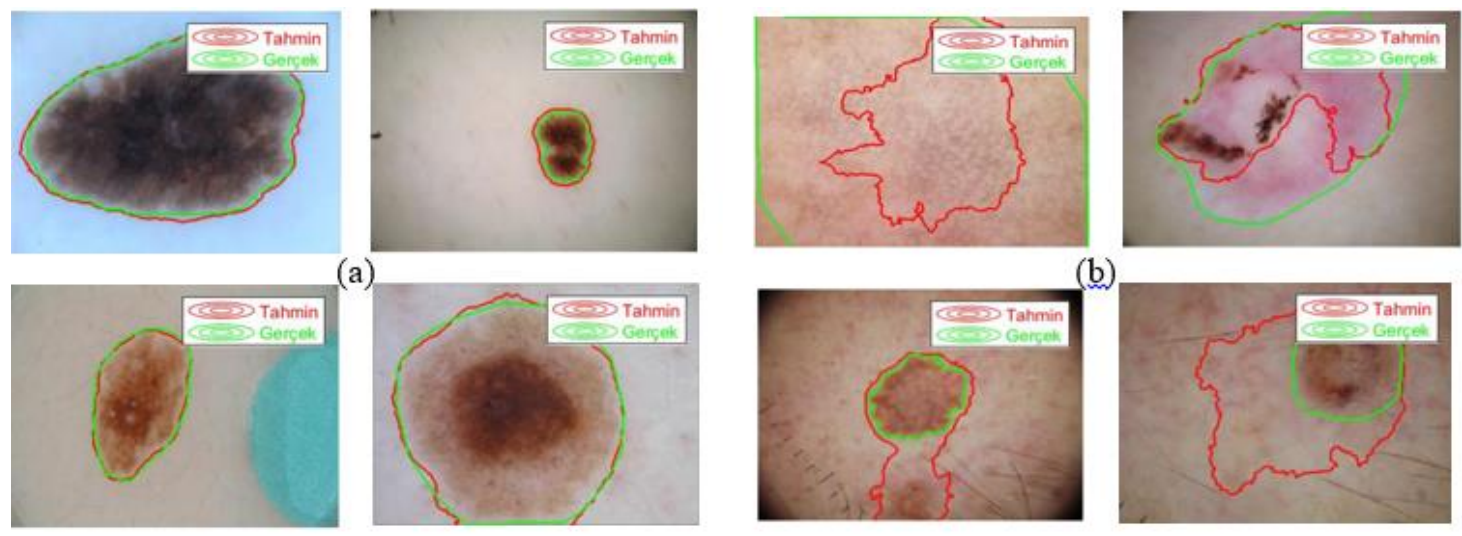

Şekil 5: (a) Doğru öğrenme, (b) yetersiz veya aşırı öğrenme durumları.

Tablo 2'de elde edilen sonuçların kesinlik, doğruluk, hassasiyet, Dice ve Jaccard skorları verilmiştir. Tablo 2 de bulunan VB, veri çoklama ve OnIs, önişlemeyi ifade etmektedir. Tablo 2'de birinci satır; eğitim görüntüleri üzerinde veri çoklama ve önişleme adımları uygulanmadan sadece eğitim görüntüleri ile eğitilmiş SegNet ağı ile elde edilen başarım sonuçlarını, ikinci satır ve üçüncü satırlar sırası ile sadece veri çoklama uygulanmış bir eğitim seti ile eğitilmiş SegNet ağı ile veri çoklama ve önişleme adımları uygulanmış eğitim görüntüleri ile eğitilmiş SegNet ağı ile elde edilen başarım sonuçlarını ifade etmektedir.

Tablo 2. ISBI2016 veri kümesi için bölütleme sonuçları

\begin{tabular}{|l|c|c|c|c|c|}
\hline & Doğruluk & Hassasiyet & Dice & Jaccard & Özgüllük \\
\hline SegNet & 0,907 & 0,846 & 0,823 & 0,732 & 0,911 \\
\hline VB+SegNet & 0,930 & $\mathbf{0 , 9 0 0}$ & 0,871 & 0,792 & 0,915 \\
\hline VB+OnIs+ SegNet & $\mathbf{0 , 9 3 5}$ & 0.879 & $\mathbf{0 , 8 8 4}$ & $\mathbf{0 , 8 0 6}$ & $\mathbf{0 , 9 3 2}$ \\
\hline
\end{tabular}

Karşılaştırma için ISBI2016 veri kümesi kullanılmıştır. Yöntemimizin diğer yöntemlerle kıyasla performans sonuçları Tablo 3'te gösterilmiştir.

Tablo 3. ISBI2016 veri kümesinde elde edilen sonuçların literatürle kıyaslanması.

\begin{tabular}{|l|l|l|l|l|l|}
\hline Yöntem & Jaccard & Dice & Hassasiyet & Özgüllük & Doğruluk \\
\hline MSCA [15] & 66.19 & 75.88 & 78.30 & 91.31 & 85.68 \\
\hline SSLS [23] & 57.20 & 69.97 & 70.04 & 97.31 & 84.67 \\
\hline GC [24] & 35.71 & 50.65 & 70.50 & 75.72 & 71.61 \\
\hline RW [24] & 42.81 & 57.52 & 72.31 & 77.07 & 74.28 \\
\hline Önerilen Y. & $\mathbf{8 0 . 5 9}$ & $\mathbf{8 8 . 4 3}$ & $\mathbf{8 7 . 8 8}$ & $\mathbf{9 3 . 2 2}$ & $\mathbf{9 3 . 4 6}$ \\
\hline
\end{tabular}

\section{Sonuç}

Son zamanlarda, özellikle beyaz ırkta melanom tipi cilt kanserinde dikkate değer oranda artış görülmektedir. Bu nedenle melanom kaynaklı ölümleri en aza indirmek için melanomun erken tanısı oldukça önemlidir. Bu çalışmada cilt lezyonlarının bölütlenmesi için derin SegNet mimarisine dayanan bir sistem geliştirilmiştir. Klasik SegNet mimarisi girişine verilen görüntülere veri çoklama ve renk tutarlılığı ve kıl silme gibi önişleme adımları uygulanarak ağın bölütleme performansı gözlemlenmiştir. Uygulanan önişleme adımları ve veri çoklama adımlarının dikkate değer oranda artırdığı gözlemlenmiştir. Bununla beraber güncel bir çalışma olan SegNet ağı ile elde edilen sonuçların güncel literatür ile yakın performans gösterdiği görülmüştür. Veri çoklama adımının derin öğrenme mimarisine dayalı SegNet'in bol miktarda veriye duyduğu ihtiyacı gidermeye yardımcı olduğu ve yetersiz öğrenme ve aşırı öğrenme gibi sorunlarla başa çıkmada önemli olduğu sonucuna varılmıştır. Gelecek çalışmalarda kodlayıcı derinliği ve farklı renk kanallarının SegNet ağının bölütleme performansı üzerindeki etkilerinin incelenmesi düşünülmektedir. 


\section{Kaynakça}

Agarwal, A., Issac, A., Dutta, M. K., Riha, K. ve Uher, V. (2017). Automated skin lesion segmentation using k-Means clustering from digital dermoscopic images. 2017 40th International Conference on Telecommunications and Signal Processing, TSP 2017 içinde (C. 2017-January, ss. 743-748). Institute of Electrical and Electronics Engineers Inc. doi:10.1109/TSP.2017.8076087

Ahn, E., Bi, L., Jung, Y. H., Kim, J., Li, C., Fulham, M. ve Feng, D. D. (2015). Automated saliency-based lesion segmentation in dermoscopic images. Proceedings of the Annual International Conference of the IEEE Engineering in Medicine and Biology Society, EMBS, 2015-Novem, 3009-3012. doi:10.1109/EMBC.2015.7319025

Ahn, E., Kim, J., Bi, L., Kumar, A., Li, C., Fulham, M. ve Feng, D. D. (2017). Saliency-Based Lesion Segmentation Via Background Detection in Dermoscopic Images. IEEE Journal of Biomedical and Health Informatics, 21(6), 1685-1693. doi:10.1109/JBHI.2017.2653179

Argenziano, G., Fabbrocini, G., Carli, P., De Giorgi, V., Sammarco, E. ve Delfino, M. (1998). Epiluminescence microscopy for the diagnosis of doubtful melanocytic skin lesions: Comparison of the ABCD rule of dermatoscopy and a new 7-point checklist based on pattern analysis. Archives of Dermatology, 134(12), 1563-1570. doi:10.1001/archderm.134.12.1563

Argenziano, G. ve Soyer, H. P. (2001). Dermoscopy of pigmented skin lesions - a valuable tool for early diagnosis of melanoma. Lancet Oncology, 2(7), 443-449. doi:10.1016/S1470-2045(00)00422-8

Badrinarayanan, V., Kendall, A. ve Cipolla, R. (2017). SegNet: A Deep Convolutional Encoder-Decoder Architecture for Image Segmentation. IEEE Transactions on Pattern Analysis and Machine Intelligence, 39(12), 2481-2495. doi:10.1109/TPAMI.2016.2644615

Barata, C., Celebi, M. E. ve Marques, J. S. (2015). Improving dermoscopy image classification using color constancy. IEEE Journal of Biomedical and Health Informatics, 19(3), 1146-1152. doi:10.1109/JBHI.2014.2336473

Bi, L., Kim, J., Ahn, E., Feng, D. ve Fulham, M. (2017). Semi-automatic skin lesion segmentation via fully convolutional networks. Proceedings - International Symposium on Biomedical Imaging, 561-564. doi:10.1109/ISBI.2017.7950583

Bi, L., Kim, J., Ahn, E., Feng, D., Fulham, M., Medicine, N., ... Hospital, A. (2016). Automated Skin Lesion Segmentation via Image-wise Supervised Learning and Multi-Scale Superpixel Based Cellular Automata School of Information Technologies, University of Sydney, Australia Sydney Medical School, University of Sydney, Australia Med-X R, 1059-1062.

Brahmbhatt, P. ve Rajan, S. N. (2019). Skin Lesion Segmentation using SegNet with Binary Cross- Entropy, 14-15.

Burg, G. (1993). Das Melanom (Serie Gesundheit) . Piper/VCH.

Chan, T. F. ve Vese, L. A. (2001). Active contours without edges. IEEE Transactions on Image Processing, 10(2), $266-277$. doi:10.1109/83.902291

Erkol, B., Moss, R. H., Stanley, R. J., Stoecker, W. V ve Hvatum, E. (2005). Images Using Gradient Vector Flow Snakes. Skin Research and Technology, 17-26.

Garnavi, R., Aldeen, M., Celebi, M. E., Varigos, G. ve Finch, S. (2011). Border detection in dermoscopy images using hybrid thresholding on optimized color channels. Computerized Medical Imaging and Graphics, 35(2), 105-115. doi:10.1016/j.compmedimag.2010.08.001

Kasmi, R., Mokrani, K., Rader, R. K., Cole, J. G. ve Stoecker, W. V. (2016). Biologically inspired skin lesion segmentation using a geodesic active contour technique. Skin Research and Technology, 22(2), 208-222. doi:10.1111/srt.12252

L.A.G. Ries, D. M. et al. (2008). SEER Cancer Statistics Review 1975-2005 . Bethesda, MD, National Cancer Institute. 13 Mart 2020 tarihinde https://seer.cancer.gov/archive/csr/1975_2005/ adresinden erişildi.

Lee, T., Ng, V., Gallagher, R., Coldman, A. ve McLean, D. (1997). Dullrazor®: A software approach to hair removal from images. Computers in Biology and Medicine, 27(6), 533-543. doi:10.1016/S0010-4825(97)00020-6

Menzies, S. W., Ingvar, C., Crotty, K. A. ve McCarthy, W. H. (1996). Frequency and morphologic characteristics of invasive melanomas lacking specific surface microscopic features. Archives of Dermatology, 132(10), 1178-1182. doi:10.1001/archderm.132.10.1178

Romero Lopez, A., Giro-I-Nieto, X., Burdick, J. ve Marques, O. (2017). Skin lesion classification from dermoscopic images using deep learning techniques. Proceedings of the 13th IASTED International Conference on Biomedical Engineering, BioMed 2017, 49-54. doi:10.2316/P.2017.852-053

Schmid-Saugeon, P., Guillod, J. ve Thiran, J. P. (2003). Towards a computer-aided diagnosis system for pigmented skin lesions. Computerized Medical Imaging and Graphics, 27(1), 65-78. doi:10.1016/S0895-6111(02)00048-4

Stolz, W., Reimann, A. ve Cognetta, A. B. (1994). ABCD rule of dermatoscopy: a new practical method for early recognition of malignant melanoma.

Tang, P., Liang, Q., Yan, X., Xiang, S., Sun, W., Zhang, D. ve Coppola, G. (2019). Efficient skin lesion segmentation using separableUnet with stochastic weight averaging. Computer Methods and Programs in Biomedicine, $178,289-301$. doi:10.1016/j.cmpb.2019.07.005

Wong, A., Scharcanski, J. ve Fieguth, P. (2011). Automatic skin lesion segmentation via iterative stochastic region merging. IEEE Transactions on Information Technology in Biomedicine, 15(6), 929-936. doi:10.1109/TITB.2011.2157829

Xie, F. ve Bovik, A. C. (2013). Automatic segmentation of dermoscopy images using self-generating neural networks seeded by genetic algorithm. Pattern Recognition, 46(3), 1012-1019. doi:10.1016/j.patcog.2012.08.012 